\title{
Construction of $\operatorname{In}_{2} \mathrm{~S}_{3} @ \mathrm{NH}_{2}-\mathrm{MIL}-68(\mathrm{In}) @ \mathrm{In}_{2} \mathrm{~S}_{3}$
}

\section{Sandwich Homologous Heterojunction for}

\section{Efficient $\mathrm{CO}_{2}$ Photoreduction}

\author{
Qingqing Hou, Xiyi Li, Yunhong Pi, Jing Xiao* \\ School of Chemistry and Chemical Engineering, and Guangdong Provincial Key \\ Laboratory of Atmospheric Environment and Pollution Control, South China University \\ of Technology, Guangzhou 510640, PR China \\ * Corresponding author: E-mail: cejingxiao@scut.edu.cn
}





\section{Supplementary Experiments}

The X-ray diffraction patterns of the optimum sample before and after reaction was recorded on PANalytical X'Pert Powder with $\mathrm{Cu} \mathrm{K} \alpha$ radiation $(\lambda=0.15406 \mathrm{~nm})$ at a voltage of $40 \mathrm{kV}$ and current of $40 \mathrm{~mA}$, respectively. The liquid products after photocatalytic $\mathrm{CO}_{2}$ reduction was analyzed by $1 \mathrm{H}$ NMR (Bruker AVANCE III HD 600). The cyclic voltammetry (CV) was measured on an electrochemical workstation (CHI660D) using $\mathrm{Pt}, \mathrm{Ag} / \mathrm{AgCl}$ electrode and glassy carbon as the counter electrode, reference electrode, and working electrode, respectively, and an electrolyte (mixed solution VTEOA: $\mathrm{V}_{\mathrm{CH} 3 \mathrm{CN}}=1: 4$, containing $0.1 \mathrm{M}$ tetra-n-butylammonium perchlorate, $\mathrm{pH}=10$, TEOA $=$ Triethanolamine). The composition and chemical states of elements in the composite were analyzed by X-ray Photoelectron Spectroscopy (XPS, ESCALAB 250Xi). 
Additional characterizations
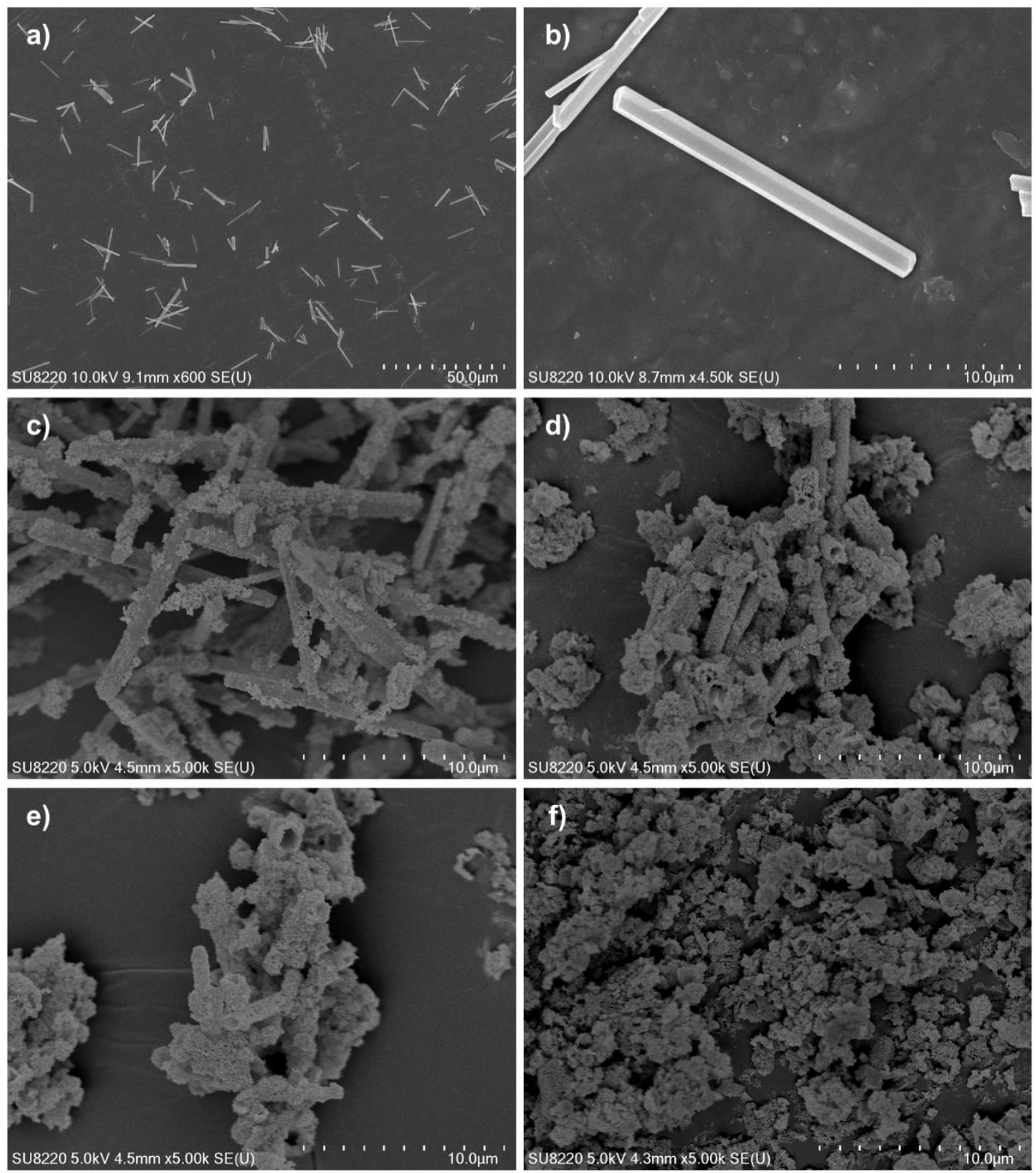

Figure S1. FESEM spectra of (a,b) $\mathrm{NH}_{2}$-MIL-68(In) and SMS(In) sandwich homologous heterojunctions collected at different sulfuration times:, (c) 2h, (d) $7 \mathrm{~h}$, (e) $12 \mathrm{~h}$ and (f) $24 \mathrm{~h}$. 


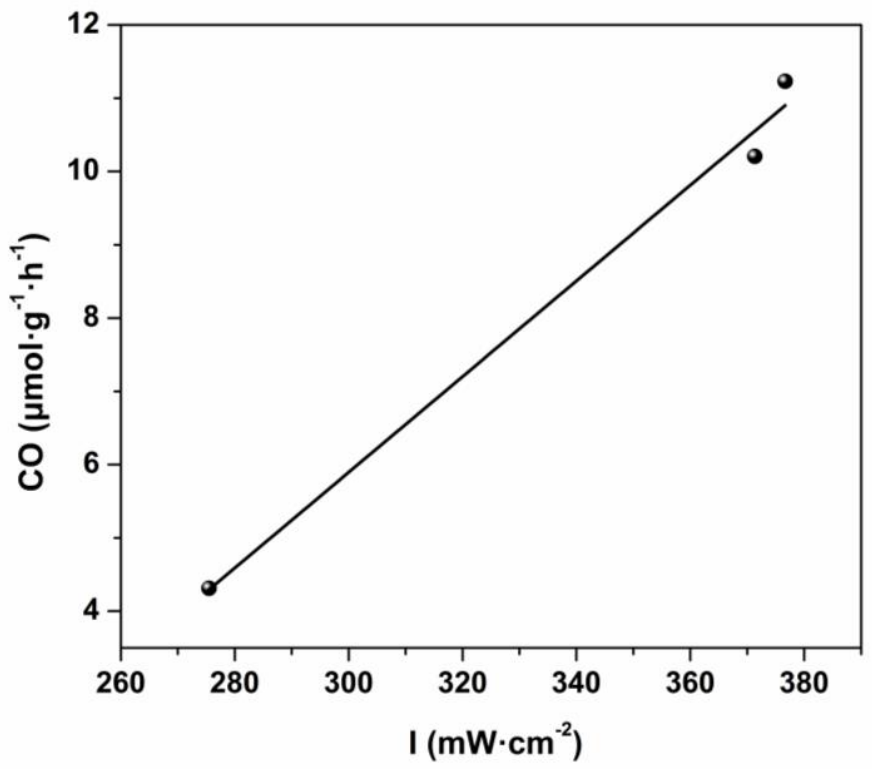

Figure S2. CO production rate of different light intensity. 


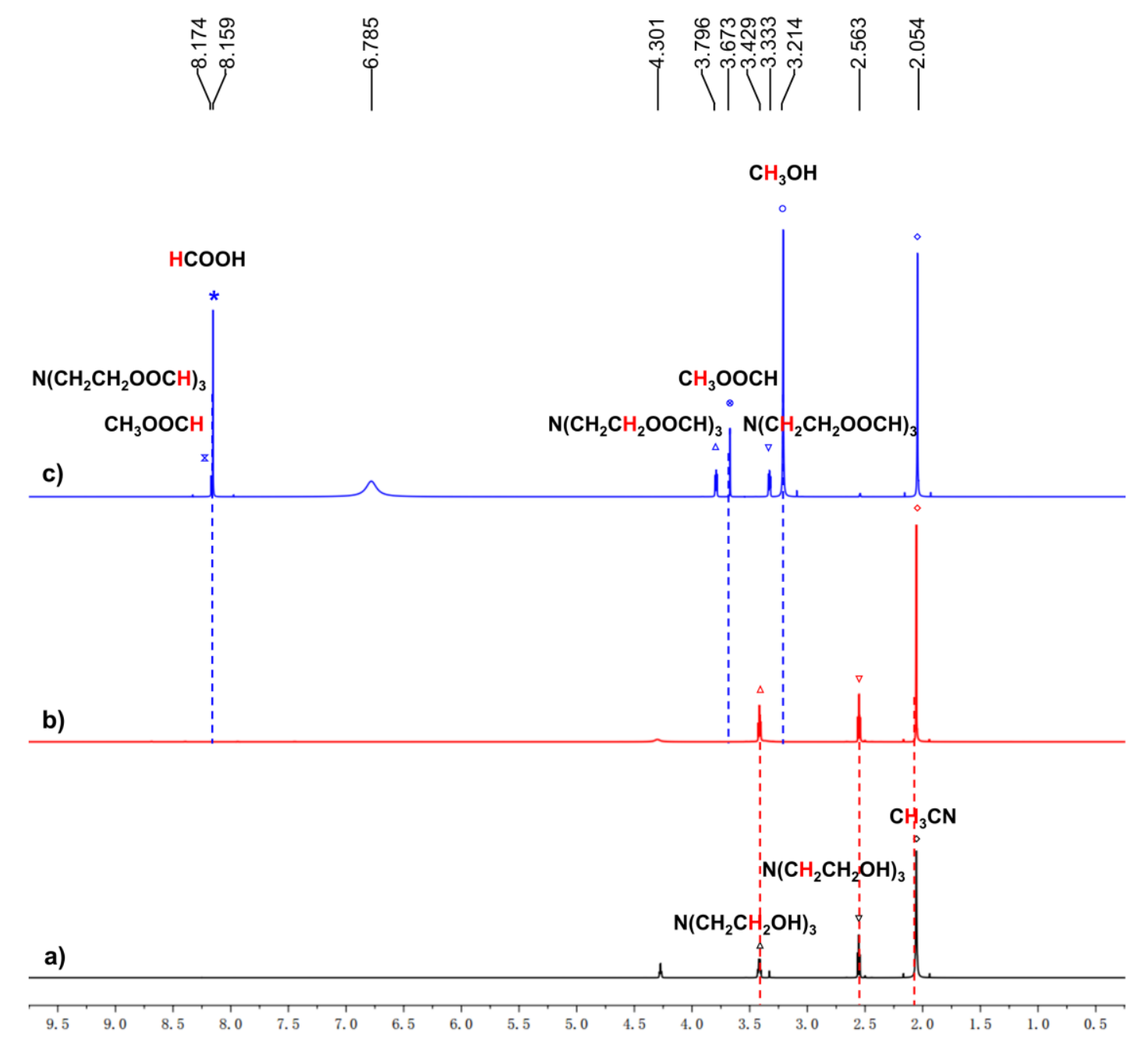

Figure S3. The $1 \mathrm{H}$ NMR Spectra of a) the mixed solution of $\mathrm{CH}_{3} \mathrm{CN}$ and $\mathrm{TEOA} b$ ) the reaction solution after reaction and c) the mixed solution of $\mathrm{CH}_{3} \mathrm{CN}$, TEOA, $\mathrm{CH}_{3} \mathrm{OH}$ and $\mathrm{HCOOH}$. 


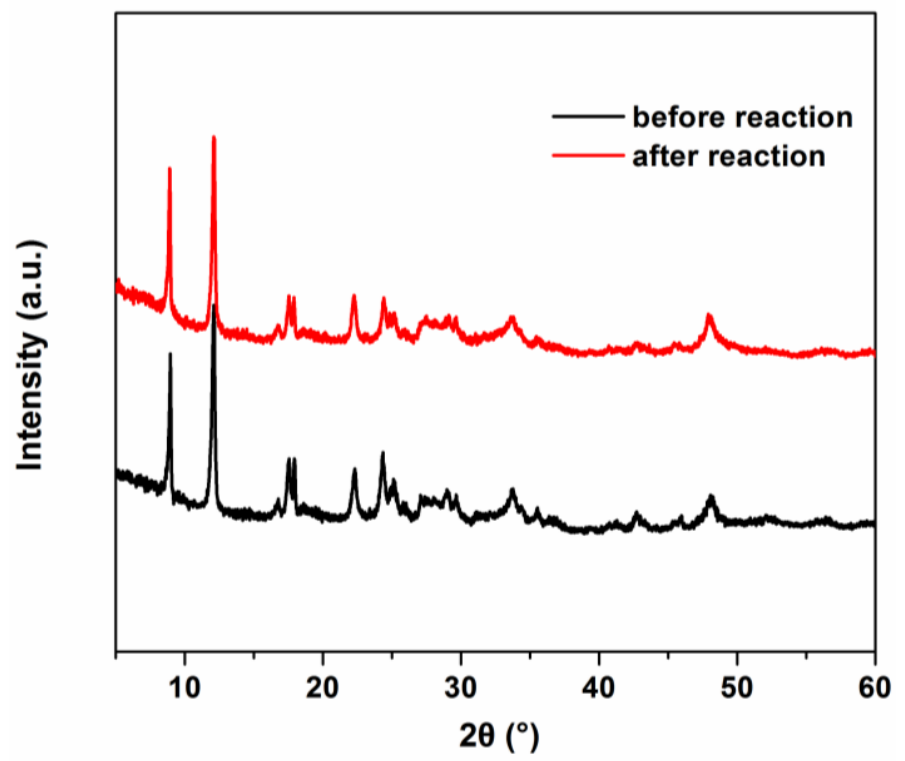

Figure S4. XRD patterns of SMS(In)-7 before and after photocatalytic $\mathrm{CO}_{2}$ reduction Reactions.

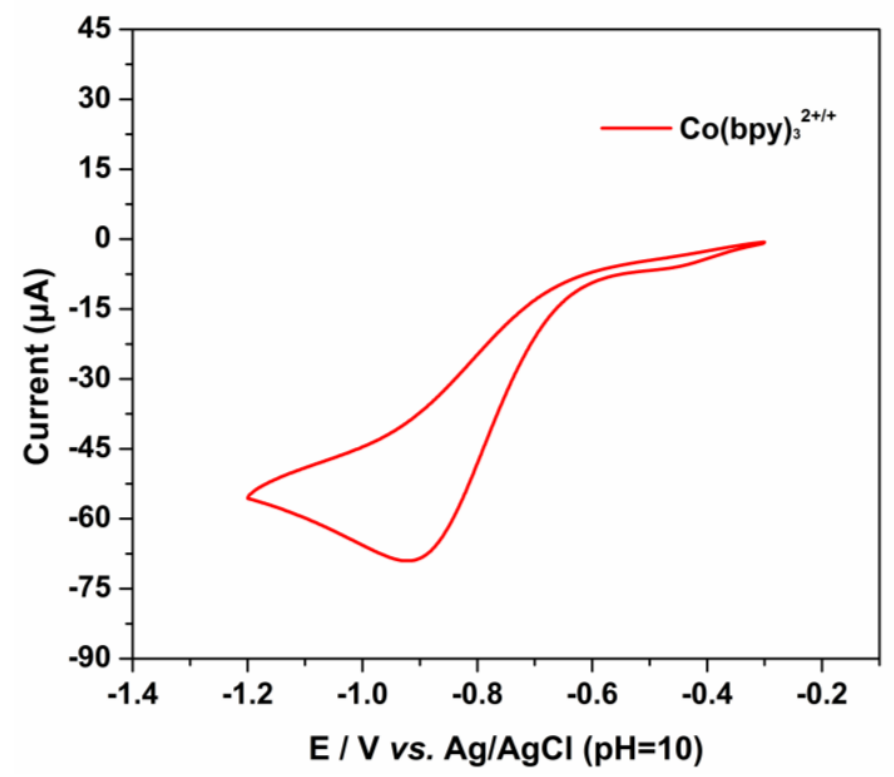

Figure $\mathrm{S} 5 . \mathrm{CVs}$ of $\mathrm{Co}(\mathrm{bpy}) 3^{2+/+}$ under $\mathrm{N}_{2}$ atmosphere in $0.1 \mathrm{M}$ tetra-n-butylammonium perchlorate in $\mathrm{TEOA} / \mathrm{CH}_{3} \mathrm{CN}$ mixed solution. 

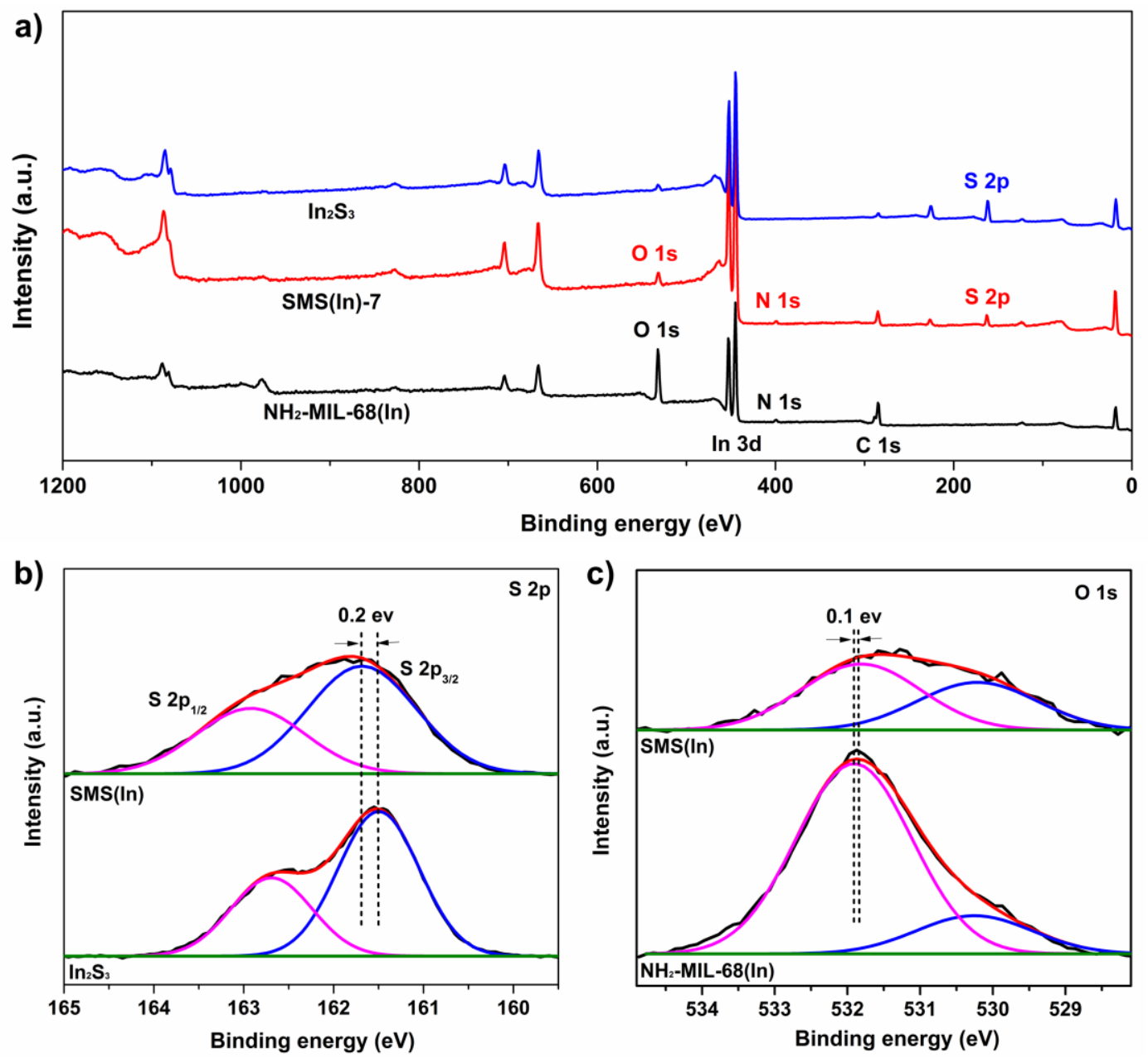

Figure S6. XPS spectra of $\mathrm{NH}_{2}$-MIL-68(In), $\mathrm{In}_{2} \mathrm{~S}_{3}$ and $\mathrm{SMS}(\mathrm{In})$ : (a) survey spectrum, high resolution XPS spectra of (b) S 2p and (c) O 1s. 

Table S1. The comparison of some typical systems

\begin{tabular}{|c|c|c|c|c|c|}
\hline \multirow[b]{2}{*}{ Photocatalyst } & \multicolumn{5}{|c|}{ Main Product } \\
\hline & Sacrificial & $\begin{array}{c}\text { Temperature } \\
\text { (K) }\end{array}$ & $\begin{array}{c}\text { Yield } \\
\left(\mu \mathrm{mol} \mathrm{g}{ }^{-1} \mathrm{~h}^{-1}\right)\end{array}$ & $\begin{array}{c}\text { Selectivity } \\
(\%)\end{array}$ & Ref \\
\hline SMS(In)-7 & TEOA & 288 & $\mathrm{CO}(11.23)$ & - & $\begin{array}{l}\text { This } \\
\text { work }\end{array}$ \\
\hline $\mathrm{P} 25$ & - & Room T & $\mathrm{CH}_{4}(0.19)$ & - & 1 \\
\hline $\mathrm{CdS}-\mathrm{TiO}_{2}-\mathrm{NS}-60^{\text {a }}$ & - & Room T & $\mathrm{CO}(3.60)$ & 87 & 2 \\
\hline Zn-MOF@GQD ${ }^{b}$ & TEOA & Room T & $\mathrm{CH}_{4}(20.9)$ & 85 & 3 \\
\hline $\mathrm{Cu}_{3}(\mathrm{btc})_{2}$-core $\mid \mathrm{TiO}_{2}$-shell & - & 313 & $\mathrm{CH}_{4}(2.64)$ & 100 & 4 \\
\hline $\mathrm{CD} @ \mathrm{NH}_{2}-\mathrm{UiO}-66$ & TEOA & Room T & $\mathrm{CO}(16.60)$ & - & 5 \\
\hline UiO-66/CNNS & TEOA & Room T & $\mathrm{CO}(9.90)$ & - & 6 \\
\hline $\mathrm{CsPbBr}_{3} @ \mathrm{ZIF}-67$ & $\mathrm{H}_{2} \mathrm{O}$ & Room T & $\mathrm{CH}_{4}(29.63)$ & 100 & 7 \\
\hline $\mathrm{g}-\mathrm{C}_{3} \mathrm{~N}_{4}$ & $\mathrm{H}_{2} \mathrm{O}$ & - & $\mathrm{CH}_{4}(0.74)$ & 86 & 8 \\
\hline $\mathrm{CdS}$ & $\mathrm{H}_{2} \mathrm{O}$ & - & $\mathrm{CO}(1.6)$ & - & 9 \\
\hline $\mathrm{ZnO}$ & $\mathrm{H}_{2} \mathrm{O}$ & 293 & $\mathrm{CO}(3.814)$ & - & 10 \\
\hline
\end{tabular}




\section{Supplementary references}

(1) Dimitrijevic, N. M.; Vijayan, B. K.; Poluektov, O. G.; Rajh, T.; Gray, K. A.; He, H.; Zapol, P., Role of Water and Carbonates in Photocatalytic Transformation of $\mathrm{CO}_{2}$ to $\mathrm{CH}_{4}$ on Titania. J. Am. Chem. Soc. 2011, 133 (11), 3964-3971.

(2) Pan, X.; Xu, Y.-J., Graphene-Templated Bottom-up Fabrication of Ultralarge Binary CdS- $-\mathrm{TiO}_{2}$ Nanosheets for Photocatalytic Selective Reduction. J. Phys. Chem. C 2015, 119 (13), 7184-7194.

(3) Wei, D.; Tang, W.; Gan, Y.; Xu, X., Graphene quantum dot-sensitized Zn-MOFs for efficient visible-light-driven carbon dioxide reduction. Catal. Sci. Technol. 2020, 10 (16), 5666-5676.

(4) Li, R.; Hu, J.; Deng, M.; Wang, H.; Wang, X.; Hu, Y.; Jiang, H.-L.; Jiang, J.; Zhang, Q.; Xie, Y.; Xiong, Y., Integration of an Inorganic Semiconductor with a Metal-Organic Framework: A Platform for Enhanced Gaseous Photocatalytic Reactions. Adv. Mater. 2014, 26 (28), 4783-4788.

(5) Li, S.; Ji, K.; Zhang, M.; He, C.; Wang, J.; Li, Z., Boosting the photocatalytic $\mathrm{CO}_{2}$ reduction of metal-organic frameworks by encapsulating carbon dots. Nanoscale 2020, $12(17), 9533-9540$.

(6) Lei, Z.; Xue, Y.; Chen, W.; Qiu, W.; Zhang, Y.; Horike, S.; Tang, L., MOFs-Based Heterogeneous Catalysts: New Opportunities for Energy-Related $\mathrm{CO}_{2}$ Conversion. $A d v$. Energy Mater. 2018, 8 (32), 1801587.

(7) Kong, Z.-C.; Liao, J.-F.; Dong, Y.-J.; Xu, Y.-F.; Chen, H.-Y.; Kuang, D.-B.; Su, C.Y., Core@Shell CsPbBr3@Zeolitic Imidazolate Framework Nanocomposite for Efficient Photocatalytic $\mathrm{CO}_{2}$ Reduction. ACS Energy Letters 2018, 3 (11), 2656-2662. 
(8) Wang, K.; Fu, J.; Zheng, Y., Insights into photocatalytic $\mathrm{CO}_{2}$ reduction on $\mathrm{C}_{3} \mathrm{~N}_{4}$ : Strategy of simultaneous B, K co-doping and enhancement by N vacancies. Appl. Catal. B: Environ. 2019, 254, 270-282.

(9) Ding, D.; Jiang, Z.; Jin, J.; Li, J.; Ji, D.; Zhang, Y.; Zan, L., Impregnation of semiconductor CdS NPs in MOFs cavities via double solvent method for effective photocatalytic $\mathrm{CO}_{2}$ conversion. J. Catal. 2019, 375, 21-31.

(10) Liu, X.; Ye, L.; Liu, S.; Li, Y.; Ji, X., Photocatalytic Reduction of $\mathrm{CO}_{2}$ by $\mathrm{ZnO}$ Micro/nanomaterials with Different Morphologies and Ratios of $\{0001\}$ Facets. Sci. Rep. 2016, 6 (1), 38474. 